\title{
Effects of the COVID-19 pandemic on college students with intellectual disability
}

\author{
Gwendolyn C. Carey ${ }^{1, *}$, Brianna J. Miller ${ }^{2}$ and Lisa A. Finnegan ${ }^{3}$ \\ Department of Exceptional Student Education, Florida Atlantic University, Jupiter, FL, USA
}

Received 18 November 2020

Accepted 7 April 2021

Pre-press 14 September 2021

Published 12 November 2021

\begin{abstract}
.
BACKGROUND: The lived experiences of adults with intellectual disability (ID) during the Coronavirus Disease 2019 (COVID-19) need to be considered when developing and implementing support for maintaining the quality of life outcomes for this population.

OBJECTIVE: This study was conducted to capture and analyze adults' lived experiences with ID during the COVID-19 pandemic.

METHODS: A qualitative study with focus groups was conducted that included two groups of adults with ID; graduated $(N=4)$ and enrolled $(N=5)$ students from a postsecondary education (PSE) program. Questions were formulated based on societal feature areas that may be affected during unplanned historical events. Data were analyzed utilizing Vivo coding.

RESULTS: Four themes (employment, daily living, social, well-being) and eleven subthemes emerged during the interviews. Participants described the impact of COVID-19, such as learning, and implementing new procedures in the workplace, taking on increased responsibilities at home, and the uncertainty of their future.

CONCLUSIONS: Findings indicated the need for support for adults with ID to be established before unplanned events to maintain their outcomes as productive members of society. Implications include the need for increased support from immediate support systems.
\end{abstract}

Keywords: Intellectual disabilities, COVID-19, vocational rehabilitation, quality of life outcomes, well-being

\section{Introduction}

As a global health crisis, the Coronavirus Disease 2019 (COVID-19) has impacted all societal sectors. The Centers for Disease Control and Prevention (CDC, 2020) stated that COVID-19 most likely spreads through airborne respiratory droplets among people who are in close contact with

\footnotetext{
${ }^{1}$ ORCID: https://orcid.org/0000-0003-4612-4730.

${ }^{2}$ ORCID: https://orcid.org/0000-0001-9857-4536.

${ }^{3}$ ORCID: https://orcid.org/0000-0001-8759-4865.

*Address for correspondence: Gwendolyn Carey, Department of Exceptional Student Education, Florida Atlantic University, Jupiter, FL 33458, USA. Tel.: +1 561799 8348; E-mail: gcarey@ fau.edu.
}

one another in enclosed spaces over an extended time. Due to the increasing likelihood of contracting the COVID-19, countries worldwide have required residents to shelter in place and restrict their exposure to the outside communities and expand the use of technology to provide social distancing for communication with non-immediate family and friends. According to the World Health Organization (WHO, 2020a; WHO 2020b), COVID19 has given rise to "... economic and social disruption ..." for adults. Along with financial security instability and social disruption, other Quality of Life Domains (QOL) including employment, physical health, psychological well-being, level of independence, social relations, environment, and 
spirituality/religion/personal beliefs (WHO, 2020c). These domains have significantly been affected for adults with intellectual disabilities (ID).

In recent years, adults with ID have increased their productivity within the above domains, based on support structures and resources for this population's needs that may increase their independence and longevity. The successes mentioned above of adults with ID require determination and hard work from the individual, but often supports and services are provided by various community agencies. Transitionaged (16-21) adults with ID typically require a high level of support to succeed in the primary life domains. In contrast, young and middle-aged adults need lower support levels (Shogren et al., 2016). Obtaining and maintaining competitive employment continues to be one of the biggest challenges facing adults with ID. Studies have indicated the need for quality of life services for this population, especially in employment, suggesting that compensatory and natural support such as employment specialists, mentors, and technology supports increased the likelihood of an adult with ID to secure successful competitive employment (Wehman et al., 2003). However, when supports and resources are scarce and depleted, the effect can worsen for adults with ID particularly when the support structures are considered significant for this population.

The COVID-19 pandemic may be a once-in-acentury event; however, adults with ID have certainly endured other disasters during the past decades. Commonalities to the hardships faced during the pandemic can be found in the experiences of adults who survived the impact and aftermath of Hurricane Katrina. Researchers found that adult survivors of Hurricane Katrina encountered extended difficulties accessing services such as transportation, healthcare, and communication (Stough et al., 2010). It was also noted that adults with ID had increased hardships securing competitive employment during the hurricane's aftermath (Stough et al., 2010). Study participants self-reported suffering from depression that they had not experienced before the hurricane. Case managers found that the support needed by adults with ID during the extended recovery effort ( 2 years after the crisis event) was more numerous and intensive than supports needed by survivors without ID (Stough et al., 2010).

It is expected that adults with ID may be more affected than their typical peers. Adults with ID face a shortage of supports and resources even during the best of times. Even when these ser- vices do exist, they are often loosely coordinated and challenging to access. Natural disasters have been shown to exacerbate these issues. According to Stough and colleagues (2016), a lack of ongoing concrete resources following Hurricane Katrina likely slowed the recovery for adults with disabilities. Of greater impact, an abundance of services and supports utilized by this population closed due to the COVID 19 virus (The Council of State Governments, 2020). Some examples of these governmental agencies and services include home and community support and services and access to education, information, steady employment, and, if needed, healthcare (The Council of State Governments, 2020).

The effects of isolation during COVID-19 can have an impact on adults with ID resilience and selfdetermination skills. Boyle et al. (2020) conducted a study and found that before Hurricane Katrina, participants in the study developed resiliency strategies for employment and natural supports to assist adults with ID within the community without those skills. These strategies and natural supports include specialized transportation, specialized services to assist with daily needs, and advocacy groups' support. Several of these resiliency strategies could potentially be implemented and enhanced when necessary during the COVID-19 pandemic.

Due to the contagious nature of COVID-19 all individuals were required to self-quarantine from all non-cohabitants causing an increase in social distancing and socialization through video conferencing platforms to communicate with others. De', Pandey, and Pal (2020) share that adults without ID have the knowledge and experience of using virtual platforms and utilizing these skills to maintain social relationships throughout the pandemic. However, if adults with ID are not taught and do not apply these technological skills, this may lead to limited socialization. Adults with ID were already more isolated than their peers, and the pandemic will likely intensify that isolation (Constantino et al., 2020).

During the pandemic, businesses in several states had to close their doors unless considered an employer of essential workers (e.g., hospital staff, public safety, first responders, and transportation). As a consequence of shuttering businesses, employers and employees alike remained uncertain about the short-term and long-term economic outcomes for adults, businesses, communities, cities, states, and nations (Altig et al., 2020). For adults with ID who were not regarded as essential workers or had the 
option of working remotely, they too found themselves unemployed and awaiting word to return to work. Health concerns also affected adults with ID returning to work. Individuals with Down Syndrome, Fetal Alcohol Syndrome Developmental Delay, or Prader-Willi Syndrome are more likely to not return to work due to health matters such as severe mobility impairment, seizure disorders or epilepsy, vision and/or hearing impairments, and other health ailments due to premature mortality (Simpson, 2015). These events will likely further exacerbate the unemployment rate for adults with disabilities.

A study examining the experiences of adults with ID in postsecondary education (PSE) programs within the U.S. identified supports such as transitioning to employment, opportunities to interact within the college setting with natural supports, and attitudes and awareness that has assisted in embedding the typical college experience for their atypical peers as necessary support elements (Dowrick et al., 2005). As these categories have decreased for this population in person due to COVID-19, providing these supports virtually can be a daunting task.

Moreover, due to financial stress, approximately one-third of the students reported that their living situation changed due to the pandemic (Healthy Minds Network, 2020). One study found that students also shared a $31 \%$ average reduction in their wages; $40 \%$ of the students indicated the loss of a job, internship, or job offer, and $61 \%$ shared the loss of income of family member (Aucejo et al., 2020).

Furthermore, the Student Experience in the Research University (SERU) Consortium at the University of California-Berkeley found that due to COVID-19, undergraduate, graduate, and professional students without ID lacked the motivation to maintain and/or improve course grades, decrease in the opportunity of face-to-face communication and dissatisfaction of transitioning remote online courses (Soria et al., 2020). In a similar study from a large university in the southwest, postsecondary undergraduate students without cognitive disabilities made decisions to delay their graduation, withdraw from classes, or switch majors due to COVID-19 and its' financial impact (Aucejo et al., 2020). It is important to note that these studies mentioned were of typically developing adult students. The lived experience findings of the impact of COVID-19 on adult students may have left out an important subgroup; adults with ID. For adults with disabilities, this is compounded by the concern that adults with disabilities are often the last group of people to potentially return or seek new employment with adults with ID impacted moreso (Erickson et al., 2014).

Perspectives from adults without ID have been shown to provide information on potential ways to aid; however, perspectives on COVID-19 for adults with ID should also be examined to consider supports and resources that can be implemented for this population. This study will answer questions related to the impact of COVID-19 on adults with ID by answering the following research questions:

(1) How has the COVID-19 pandemic affected employment for adults with ID that attend or attended postsecondary education programs?

(2) How has the COVID-19 pandemic affected social interactions for adults with ID?

(3) How do adults with ID perceive the impact of the COVID-19 pandemic on their own lives?

\section{Methods}

\subsection{Participant eligibility and selection}

Nine participants were in the focus groups. The participants consisted of current college students and graduates with ID enrolled in a postsecondary education (PSE) program located within an institution for higher education (IHE) in the United States' southeastern region. Participants were recruited based on recommendations from instructors in the PSE program. Five enrolled, and four graduates were recommended to be a part of the focus group. The IQ of participants ranged from 40 to 74 . All participants were able to communicate their wants and needs verbally. Additional demographic information can be found in Table 1.

It should be noted that all participants received instruction in self-determination and technology as part of their university programming prior to the pandemic. Participants represented sub-groups within the PSE program that may have been affected by COVID-19. Zoom meetings were held to obtain consent and assent. Before each Zoom meeting, consent and assent forms were emailed to the participant's legal guardian. Because the participants had legal, authorized guardians, each participant's legal guardian was provided a consent form. The legal guardian signed the consent form. After consent was received from the legal guardian, a researcher met with all participants to inform them of the study and present the adult assent form. All participants 
Table 1

Participant demographics

\begin{tabular}{lcclllc}
\hline Participant name & Sex & Age & Living arrangements & Employment status & WAIS IV & Disability diagnosis \\
\hline Enrolled students & & & & & & \\
\hline Beyonce & F & 21 & Group home & Not employed & 60 & ID \\
Bobby & M & 23 & Independent & Not employed & 66 & ID, ASD \\
Coral & F & 40 & Lives with family & Employed & 74 & ID \\
Auggie & F & 20 & Lives with family & Employed & 60 & ID, ASD \\
Michael & M & 26 & Lives with family & Not employed & 74 & ID, ASD \\
Graduated students & & & & & & Not available \\
Matilda & F & 31 & Semi-independent & Not employed & 40 & ID \\
John & M & 24 & Lives with family & Employed & ID \\
Diana & F & 26 & Lives with family & Employed & 49 & ID \\
Belle & F & 28 & Lives with family & Employed & 53 & ID \\
\hline
\end{tabular}

*WAIS IV: Wechsler Adult Intelligence Scale - Fourth Edition.

were read an assent for individuals with cognitive impairments on Zoom. All participants provided verbal and written assent. All consent and assent forms were signed and emailed to the researcher. The study was approved by Florida Atlantic University's Institutional Review Board (1634439-1) prior to the implementation of the focus groups.

\subsection{Setting}

Due to COVID-19 and the restrictions pertaining to social distancing, the participants, facilitator, and notetaker conducted the focus groups on Zoom. As the result of ample opportunities to practice Zoom meetings via previous synchronous online courses in the PSE program, participants were familiar with Zoom. Researchers were also familiar with Zoom from the prior teaching of courses and communication with colleagues using Zoom. Participants were asked to $\log$ on to the meeting 5-10 minutes before troubleshooting any technical computer, web camera, and microphone difficulties.

\subsection{Development and implementation of questions}

Researchers initially developed questions based on three core components that COVID-19 may have affected, provided by the Center for Disease Control and Prevention (CDC), employment, daily living outcomes, and socialization (Center for Disease Control and Prevention, 2020). The researchers also searched for qualitative studies within the past ten years and found that the above core components are similar or equivalent. Open-ended questions within each core component were formulated based on previous qualitative research on how historical events impacted the human experience for individuals with ID (Peek \& Stough, 2010; Stough et al., 2016).

Before administering the focus group questions to participants, the researchers analyzed the questions to decrease participant comprehension difficulties. The researchers distributed the focus group questions to three university faculty members and one special education (SPED) doctoral student. Questions were disseminated via email to the adults. The results indicated that the terminology was proficient and on a third-grade level for participants to understand, and no modifications to the questions were needed. The results also indicated an additional category and questions added based on the correlation between COVID-19 and self-care. Therefore, the researchers developed the category well-being along with three related questions. See Table 2 for the semi-structured interview questions.

\subsection{Experimental design}

Researchers used the qualitative method to capture sub-themes from the participant's responses. Using a qualitative approach, this design may decrease hasty answers from participants and increase elaboration on questions that may not be optimized using a survey/scale.

\subsection{Procedure}

Participants were notified two weeks in advance of when the focus group session would occur on Zoom. Participants were divided into two groups: enrolled students and graduated students from the PSE program. Each participant focus group was provided with a Zoom link and the password to enter the Zoom meeting. For each group meeting, the facilita- 
Table 2

Semi-structured interview questions

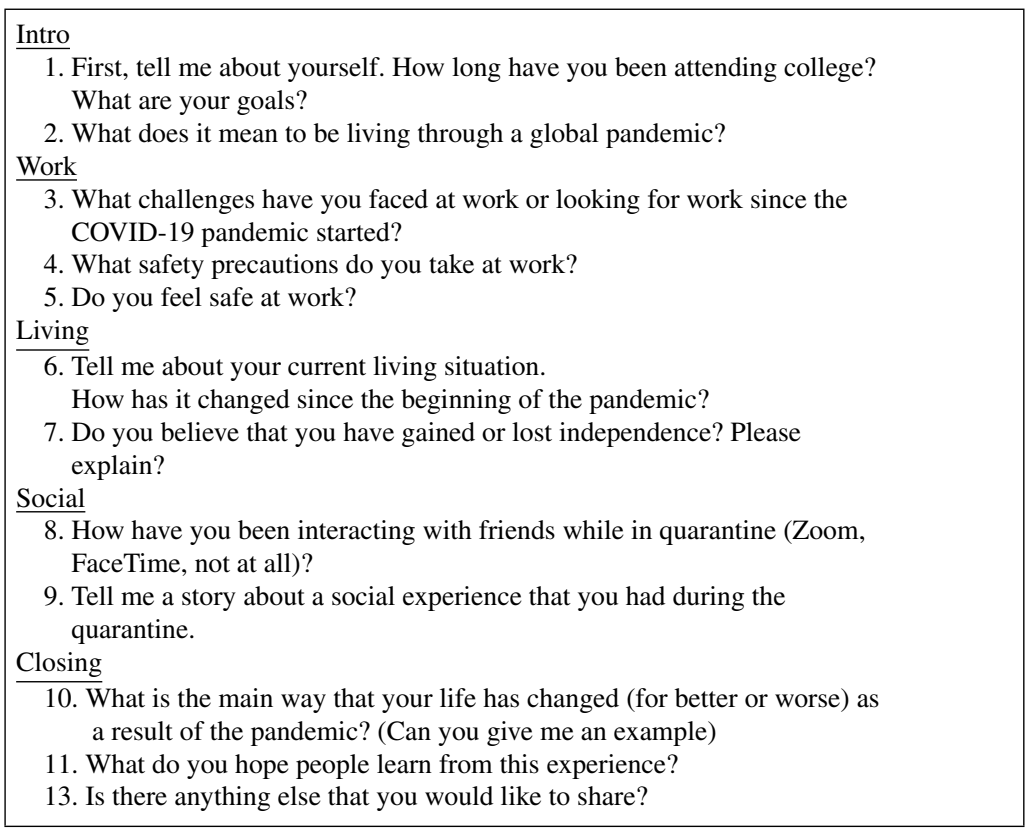

tor and the notetaker joined the meeting 15 minutes before to discuss any clarification components of the focus group (e.g., facilitator probing students to give examples and elaborate on brief responses). The facilitator and the notetaker greeted the participants and discussed online expectations. For example, "Unless you are talking, please mute your microphone" and "When you want to answer, comment, or ask a question, make sure that you unmute your microphone." The facilitator also reminded the participants that the meeting would be recorded.

\subsection{Implementation of focus group}

The notetaker initiated the Voice Recorder app on their iPhone and proceeded to ask questions to the focus group. The focus group discussions allowed the participants to have an open dialogue on the effects of COVID-19 in their lives. The notetaker also took notes on participant responses, body language, and tone. At the end of the focus group, the facilitator and the notetaker thanked the participants for participating in the focus group.

\subsection{Data collection}

Recordings were collected using a voice recorder app on the iPhone Voice Recorder app. Researchers researched various apps and found that the Voice
Recorder app for iPhone provides a software setting that enables a feature to record verbal language from adults via Zoom. Prior to each focus group, the researcher/facilitator tested the app by registering themselves, playing back the recording, and ensuring that the Zoom record enable-feature was within the app's settings.

\subsection{Coding data}

The focus group sessions were screened and audio recorded to analyze sub-themes from the focus group data. The researcher screen recorded the Zoom meetings (recording permission was stated in the consent and assent forms).

\subsection{Data analysis}

The researchers used Saldana's (2015) guidelines, Coding Manual for Qualitative Researchers to analyze the data. In Vivo coding was utilized throughout data analysis. Themes and subthemes were determined based on coding patterns from participant verbal indicators correlated with "unity and multiplicity" (p. 5). The researchers also found responses with similar meanings that corresponded with a theme or subtheme. Researchers used affective methods within the focus group questions, subjectivity perceptions, attributes of their experience with 
COVID-19, and used descriptive coding to insert participant responses in designated themes and subthemes.

\section{Findings}

The data collected was analyzed and organized into four major themes (e.g., employment, daily living, social, and well-being.) Each major theme was then divided into sub-themes creating a total of eleven subthemes. There was a great deal of similarity between the current student group and the graduated group's responses. However, some themes and sub-themes were mentioned more often by one group than the other. Table 3 displays the quotes of participants organized by theme and subtheme.

\subsection{Employment}

\subsubsection{New precautions in the workplace}

Participants in both the PSE college graduated and enrolled college student focus groups shared stories of how their jobs have changed due to the COVID-19 pandemic. These changes varied from increased procedures, including temperature checks and screenings that are now required before an employee can even enter their place of employment, to new or modified tasks such as sanitizing shopping carts or quarantining books for 72 hours an employee completes during their workday. One participant from the current student group shared that she is currently working from home. She stated that all items she needs to complete her assigned tasks are delivered to her house weekly.

\subsubsection{Preparing for the future}

Adults from both focus groups expressed using their time during quarantine to increase their skills and prepare for the future. Participants from both groups sought continuing education, including taking a fully online, asynchronous computer applications course offered by the local state college to complete a virtual training program that ended with the participant becoming a fully certified yoga instructor. A graduated participant shared the story of continuing her job search, interviewing, getting hired, and beginning a new job, all in an entirely virtual environment.

\subsubsection{Job search on hold}

Several participants shared they temporarily put their job searches on hold. Those adults expressed concerns regarding health and safety. They stated they were not comfortable being out in the community to visit potential employers while the positivity rate for COVID-19 was still high in their state. This sub-theme was more common among participants in the current student group than those who had already graduated from college.

\subsection{Daily living}

\subsubsection{Increased responsibilities}

The enrolled participants stated an increase in responsibilities at home than the graduated participants. Various tasks included everyday household chores, including laundry, dishes, and meal preparation. A few of the functions mentioned appear to be of heightened importance during the time of a pandemic. One participant is now responsible for ensuring that all family members take their daily vitamins. This trend was minimally noted for participants in the graduated group.

\subsubsection{New worries}

Both current students and graduates expressed that their days are often filled with more worries than before the pandemic. These worries range from worrying about oneself, family, and friends to concerns for society in general. A pair of graduated siblings shared concerns about their elderly grandmother not being able to visit in person for her birthday. One participant expressed that he was worried about his own life and was afraid that he would potentially contract the virus and die. Living through a pandemic is a new experience for everyone. This was a common thread among every participant in the study. Worries were one of the two most mentioned topics by both groups.

\subsection{Social}

\subsubsection{New virtual opportunities}

Participants from both groups reported shifting their social lives from in-person activities to virtual interactions. These virtual interactions ranged from 1:1 day-to-day socialization activity to formal group gatherings. One participant described in detail the virtual cooking experiences he participated in with his former peer mentor. The pair cooked meatballs, chicken, and other recipes together via Zoom. Another participant proudly shared with excitement the details of how she celebrated her 21st birthday party with games and well wishes via Zoom. All participants reported either limiting or eliminating 
Table 3

Participant quotes

Themes/sub-themes
Employment
New precautions in the
Preparing for the future
Job search on hold

Daily living

Increased responsibilities

New worries

Social

New virtual opportunities
Quote

I can't just walk in and go. The supervisor will come ask me a bunch of questions. They will also give me a temp check. (Coral, current student)

Clean a cart and basket. Then gotta clean it again. Then again. Got to wear a mask. Free sanitizer! Six foot away. (Diana, graduate)

Work from home. (Auggie, current student)

I got hired there, it was probably a month ago. (Auggie, current student)

I got certified! I am a fully certified yoga teacher. My yoga training class was over and then it happened. I was able to teach my first class. It was a phenomenal opportunity. (Bobby, current student)

PBSC, I'm doing the business certificate career. They switched everything online. (Matilda, graduate)

No, I told my VR counselor that I'm holding off. (Matilda, graduate)

I think I will stay in the house because the pandemic is controlling our state. (Bobby, current student)

No, no! (Michael, current student)

It's my job to make sure that all three of us take our vitamins. (Coral, current student)

I wash my mask every time. (Bobby, current student)

To be honest, I like my apartment because I can cook, I can bake, I can do my laundry. (Bobby, current student)

I do the laundry. I do it all by myself. (Belle, graduate)

If I got it, I would possibly die and I don't want to die. (Bobby, current student)

There is a lot of people are dying and kids too and I feel bad for them. (Belle, graduate)

It changed my life, because I worry about my great grandma. She is getting old. (John, graduate)

I guess it's just never been through COVID-19 before. (Diana, graduate)

People I see go into places without even warning a mask or anything, so I think they could be exposed, because if they don't wear a mask they can be sick, they are going to have a high fever and a cough and everything. (Matilda, graduate)

I hope that people learn how to be safe. (Bobby, current student)

I did my virtual party on Thursday on Zoom. It was a lot of people that came. (Auggie, current student)

I watched church service online. (Coral, current student)

We cook on Zoom. He teach me to cook. Something unique. Cooking meatballs is so delicious. (Bobby, current student)

I did book club online. It was Zoom. (Matilda, graduate)

The biggest thing my life was changed when I do more virtual activities than in person activities. (Michael, current student)

Creative family time

We make dinner and socialize. We do dinner and a movie. (Coral, current student)

I do racing with dad. (Diana, graduate)

We have barbecues. (Beyonce, current student)

We go out and have lunch and do stuff like that. (Matilda, graduate)

We watch movies, we play games, we eat! We had an ice cream party at the group home.(Beyonce, current student)

Missing everyday life

I miss going to class. (Auggie, current student)

I hope we can go back to campus. I miss the dining hall and all the food options. (Michael, current student)

I think I haven't seen my girlfriend since March. It's been hard on us. She Facetimes me every week because she cares about me. (Bobby, current student)

I don't like to stay home, but I have to stay home because of the COVID-19 virus. (Auggie, current student)

Miss going to work. To make some money. (Belle, graduate)

Well-being

I miss Special Olympics and other activities. They just stopped. I open it reopens soon, but I don't know when. (Michael, current students)

Intentional exercise

I try to go for a walk. In fact, I took a walk this morning. I'll usually go for a walk around my neighborhood. (Coral, current student)

Zumba and Pump! We go on Facebook and do it in the kitchen. (Belle, graduate)

I do work out with him at the gym. (Diana, graduate)

Improved nutrition

People need to be healthy and they need to eat good food to be healthy.

I am eating a lot of fruits and vegetables. (Belle, graduate)

I have MyFitnessPal. I track my fruit that way. (Matilda, graduate)

Every morning, my mom, she gave us a healthy protein shake. (John, graduate)

I went to therapy. (Matilda, graduate)

I did yoga for fun and for mental health. I relax and do my Chi Gon, and I feel better. (Bobby, current student) 
in-person social activities. Adults reported being both participants and initiators of these virtual social activities.

\subsubsection{Creative family time}

Both groups reported spending increased amounts of time with the people with whom they live. This includes immediate family members, support personnel, and group home peers, depending on the individual's living situation. A variety of newly initiated family activities were shared. One participant shared that she and her parents enjoyed weekly dinner and movie nights while staying safe during quarantine at home. Participants reported having mixed feelings about this additional time with their family and peers. Some enjoyed the additional time to bond, while others felt frustrated by their peers' extra time.

\subsubsection{Missing everyday life}

The majority of participants from both groups expressed missing various aspects of daily life prior to the pandemic. A variable that all of the participants in both focus groups discussed the absence of socialization which included extended family and peers, activities, routines related to college, work, and social aspects of life. One participant expressed sadness about not attending classes on campus and seeing her friends in person. While another participant shared, he missed the dining options provided on a college campus. Participants expressed an eagerness for a return to normal.

\subsection{Well-being}

\subsubsection{Intentional exercise}

Participants from both groups shared their efforts to engage in exercise during the quarantine. Exercise activities included both indoor and outdoor activities, as well as in-person and virtual fitness experiences. A participant from the graduated group reported that they go to the gym with their brother regularly. Two participants shared that they have been taking virtual Zumba ${ }^{\circledR}$ and Pump classes together. Several participants reported going for regular walks within their neighborhoods. Walking was the most common noted type of exercise.

\subsubsection{Improved nutrition}

Both groups discussed the importance of making good nutritional choices to increase overall health during the pandemic. Participants shared the need to eat healthy foods such as fruits and protein. One participant mentioned utilizing an app to help her track her intake of food. Although concern for nutrition was observed across groups, it was more frequently mentioned by those in the graduated group.

\subsubsection{Self-care}

Both groups discussed the importance of self-care during these challenging times. Participants reported engaging in self-care activities. Self-caring activities, including participation in virtual yoga and Chi Gong classes to reduce stress, attending in-person therapy sessions, and following health guidelines by wearing masks and social distancing.

\section{Discussion}

This study aimed to discover and share the impact of the COVID-19 pandemic on adults with ID. All participants in this study are attending or have graduated from a PSE at an IHE. The results show that participants were impacted in the employment, independent living, and social-emotional domains. The fact that all participants had intense and direct instruction in the areas of self-determination and technology before the start of the pandemic may have provided them an advantage over adults with ID who had not participated in higher education.

Regarding employment, participants were impacted in various areas. Some chose to delay all employment-related activities until the pandemic concludes. Others used the time in quarantine to learn new work-related skills. Participants already employed adapted to new health and safety procedures in the workplace. The variation in effects may partly result from where any given participant was in the employment process. Those already employed were willing to make the necessary changes required to maintain employment.

The daily living domain was also impacted, for the majority of participants reported taking on both increased responsibility in their homes and increased levels of worry and stress. These increased responsibilities and concerns likely led to the unintended central theme of well-being discovered during data analysis. Participants shared their strategies for both physical and emotional well-being.

All participants reported significantly decreasing or eliminating in-person social activities. However, participants switched many of their previous activ- 
ities from in-person to virtual and even began engaging in some new activities utilizing virtual platforms. Participants were often the ones initiating and coordinating the above-mentioned virtual activities. This is likely connected to the overwhelmingly common theme of missing everyday life. Participants overcame obstacles and utilized problem-solving skills to connect with familiar people and activities during these unfamiliar times.

Findings in this study can be correlated to the importance of adults with ID developing selfdetermination. That can be observed in the broad range of outcomes. Additionally, adults with ID are often left out of the literature when research topics begin to diverge from special education. These adults deserve to have their voices shared during this unique and defining moment in history.

Participants expressed a variety of changes related to work settings and job searchers. Continued progress toward successful, competitive employment can be made by vocational coordinators, job coaches, and employers reaching their clients and employees with ID to determine any additional supports that may be needed due to the COVID-19 pandemic. Stough \& Sharp (2010) expressed that support for adults with disabilities is essential to be more in-depth and continue for an extended time during a crisis.

The most prevalent theme shared by numerous participants was worrisome. Therefore, it is strongly recommended that adults with ID are provided the necessary mental health resources required to manage stress and anxiety during a crisis, which creates worry and isolation (Boyle et al., 2020).

\section{Implications for practice}

The findings of this study indicated reevaluating supports and resources for adults with ID in unforeseen historical events. Modeling and applying supports such as teaching technological skills via inperson and virtually may decrease the difficulties of transitioning from in-person supports to virtual vocational rehabilitation and supports and resources from a PSE program. Colleges and universities can support adults with ID by providing instruction in technology to assist with virtual connections. This study's present findings suggest that adults with ID would benefit from additional specialized, temporary support from universities, employers, and community agencies. Employers should also explore flexible work options for their employees with ID. Community agencies such as Vocational Rehabilitation and Agency for Persons with Disabilities can assist adults with ID in connection to and paying for mental health services related to developing coping strategies. These strategies will assist adults with ID to build resilience against the pandemic's effects (Boyle et al., 2020). Support providers for this population must evaluate the requisite areas that adults without ID can navigate to adjust and/or modify avenues and circumstances that may arise during and after unforeseen events. These requisite skills should be taught with needed supports to adults with ID. Service providers and other care providers should also implement proactive procedures that will be beneficial to this population. These procedures may include, but not limited to, assessing employment and communication deficits that are necessities and will need to be taught and applied through contrived and spontaneous situations. For example, if the client has difficulty using video conferencing to communicate needs to an employer, a visual and/or written task analysis with a certain measure on gaps within this area. Once the client has met the suggested criterion measure that was set, opportunities for maintenance and generalization must be established with continuous progress monitoring of the process and the provision of extraneous supports e faded or eliminated when the objective is fully demonstrated. Also, using natural environment supports to illustrate executive functioning skills such as self-regulation and self-monitoring skills that can be used in the workplace is needed for generalization and can be less stigmatizing (Dowrick et al., 2005; Erickson et al., 2014). Preparing the client for impactful changes that may affect their probability of employment or maintaining their job may decrease the client's frustration and anxiety (Constantino et al., 2020; Peek \& Stough, 2010).

\section{Implications for research}

A replication of this study is recommended to determine the impact of the COVID-19 pandemic on adults with ID who have not attended this IHE. The conditions of the study can be easily recreated with various participant groups. A comparison between the impact of the COVID-19 pandemic on adults with ID and adults without ID would also add to the literature, examining other supports and resources that were not addressed in this study. 


\section{Limitations and future research}

First, all participants were graduates of or current college students in similar PSE programs within an IHE. However, this study does not focus on other COVID-19 during and after-effects from other PSE programs or community programs that cater to adults with ID. Although efforts were made by the researchers to promote diversity in the focus groups, generalization beyond this population is not yet known. Second, participants in this study had an abundance of opportunities to demonstrate their PSE program's technological capabilities with decreased technical issues. This could be a limitation for adults within this population who are not proficient in technology and video conferencing software. Third, not all participants were employed or employed, making it difficult for some participants to elaborate on their employment experiences and COVID-19. The familiarity of the researcher and notetaker may contribute to the participants' willingness to answer questions in-depth. The willingness to elaborate on questions may not be the instance for a focus group with an unfamiliar facilitator and notetaker.

\section{Conclusion}

When determining designated supports and resources during an unplanned historical event, all populations need to be considered in the planning, including adults with ID. Adults with ID face the same challenges as their peers without disabilities. However, those with ID are more likely to need intensive, extended supports to recover from unplanned societal events.

\section{Acknowledgment}

None to report.

\section{Conflict of interest}

The authors declare that they have no conflict of interest.

\section{Disclaimer}

All human subjects within this study are in accordance with the Committee on the Human Exper- imentation of the institution in which the experiments were done or in accordance with the Helsinki Declaration of 1975 .

\section{Ethics approval}

Ethical approval for this study was obtained from Florida Atlantic University's Institutional Review Board (1634439-1).

\section{Funding}

None to report.

\section{Informed consent}

Written informed consent was obtained from the guardians of participants prior to the study. Written assent was obtained from all participants prior to the study.

\section{References}

Altig, D., Baker, S., Barrero, J. M., Bloom, N., Bunn, P., Chen, S., Davis, S. J., Leather, J., Meyer, B., Mihaylov, E., Mizen, P., Parker, N., Renault, T., Smietanka, P., \& Thwaites, G. (2020). Economic uncertainty before and after the COVID-19 pandemic. Journal of Public Economics, 191, 1-13, https://doi.org/10.1016/j.jpubeco.2020.104274

Aucejo, E. M., French, J., Araya, M. P. U., \& Zafar, B. (2020). The impact of COVID-19 on student experiences and expectations: Evidence from a survey. Journal of Public Economics, 191, 1-15 https://doi.org/10.1016/j.jpubeco.2020.104271

Boyle, C. A., Fox, M. H., Havercamp, S. M., \& Zubler, J. (2020). The public health response to the COVID-19 pandemic for people with disabilities. Disability and Health Journal, 13(1). https://doi.org/10.1016/j.dhjo.2020.100943

Center of Disease Control and Prevention. (2020). COVID-19. https://www.cdc.gov/coronavirus/2019-ncov/index.html.

Constantino, J. N., Sahin, M., Piven, J, Rodgers, R., \& Tschida, J. (2020). The impact of COVID- 19 on adults with intellectual and developmental disabilities: clinical and scientific priorities. American Journal of Psychiatry, 177(11), 1091-1093 https://doi.org/10.1176/appi.ajp.2020.20060780

De', R., Pandey, N., \& Pal, A. (2020). Impact of digital surge during Covid-19 pandemic: A viewpoint on research and practice. International Journal of Information Management, 55, 102171.

Dowrick, P. W., Anderson, J., Heyer, K., \& Acosta, J. (2005). Postsecondary education across the USA: Experiences of adults with disabilities. Journal of Vocational Rehabilitation, 22(1), 41-47. 
Erickson, W. A., von Scharader, S., Bruyere, S. M., \& VanLooy, S.A.(2014). The employment environment: Employer perspectives, policies, and practices regarding the employment of persons with disabilities. Rehabilitation Counseling Bulletin, 57(4), 195-208.

Healthy Minds Network. (2020). The impact of covid-19 on College student wellbeing. https://healthymindsnetwork. org/wp-content/uploads/2020/07/Healthy_Minds_NCHA_ COVID_Survey_Report_FINAL.pdf

Peek, L., \& Stough, L. M. (2010). Children with disabilities in the context of disaster: A social vulnerability perspective. Child Development, 81(4), 1260-1270.

Saldaña, J. (2016). The coding manual for qualitative researchers. SAGE.

Shogren, K., Wehmeyer, M., Seo, H., \& Thompson, J. (2016). Impact of the protection and advocacy subscale on the factorial validity of the support's intensity scale-adult version. American Journal on Intellectual and Developmental Disabilities, 121(1), 48-64.

Simpson, J. (2105). Common Physical Concerns. In S. Melrose, D. Dusome, J. Simpson, C. Crocker, \& E. Athens (Eds.), Supporting Individuals with Intellectual Disabilities \& Mental Illness: What Caregivers Need to Know. (pp 39- 64). BCcampus. Retrieved from http://opentextbc.ca/caregivers/

Soria, K. M., Horgos, B., Chirikov, I., \& Jones-White, D. (2020). First-generation students' experiences during the COVID19 pandemic. SERU Consortium, University of California - Berkeley and University of Minnesota. Retrieved from https://cshe.berkeley.edu/seru-covid-survey-reports
Stough, L.M., Sharp, A. N, Decker, C., \& Wilder, N. (2010). Disaster case management and adults with disabilities. Rehabilitative Psychology, 55(3), 211-220.

Stough, L. M., Sharp, A. N., Resch, J. A., Decker, C., \& Wilker, N. (2016). Barriers to the long-' term recovery of ID following a disaster. Disasters, 40(3), 387-410. https://doi.org/10.1111/disa.12161

The Council for State Governments (2020). COVID-19 and impacts on adults with disabilities. https://web.csg.org/ covid19/2020/03/31/covid-19-and-impacts-on-adults-withdisabilities/

Wehman P., Revell W.G., \& Brooke V. (2003). Competitive employment: Has it become the "first choice" yet? Journal of Disability Policy Studies, 14(3), 163-173. https://doi.org/10.1177/10442073030140030601

World Health Organization. (2020a). Impact of COVID-19 on people's livelihoods, their health and our food systems. https:// www.who.int/news/item/13-10-2020-impact-of-covid-19-onpeople's-livelihoods-their-health-and-our-food-systems

World Health Organization. (2020b). Transmission of SAVS-CoV2: Implications for infection prevention precautions scientific brief. https://www.who.int/news-room/commentaries/detail/ transmission-of-sars-cov-2-implications-for-infection-preven tion-precautions

World Health Organization. (2020c). WHOQOL: Measuring Quality of Life. https://www.who.int/healthinfo/survey/whoqol-qua lityoflife/en/ 